\title{
Intracoronary Multi-link stents: experience in 218 patients using aspirin alone
}

\author{
A L Calver, K D Dawkins, H H Gray, G A Haywood, J M Morgan, I A Simpson
}

\begin{abstract}
Objectives-To assess procedural outcome, complications, and clinical follow up in 218 patients who underwent treatment with 297 Multi-link (Guidant) stents implanted without the use of intravascular ultrasound (IVUS) or quantitative coronary angiography (QCA), and using aspirin alone as antiplatelet therapy.
\end{abstract}

Methods-The case records and angiograms were reviewed and the patients were contacted by telephone to determine their symptoms and any adverse events at follow up. Data were analysed using Fisher's exact test.

Results-Of the 218 patients included in the study, 45 had multivessel intracoronary intervention, and 55 had unstable angina. The mean (SD) length of hospital stay following the procedure was $2.0(2.1)$ days. There were two early deaths at less than 30 days, and two deaths during follow up at more than 100 days. Ten patients suffered complications during the first 30 days: four had subacute stent thrombosis, of whom two died and two were treated successfully with coronary artery bypass grafting; five had a non- $Q$ wave myocardial infarction; and one had a femoral false aneurysm. Patient outcome was analysed according to stent diameter $(3.0 \mathrm{~mm}$ or less, or $3.5 \mathrm{~mm}$ or more) and by angina status (stable or unstable). In patients in whom at least one stent was $3.0 \mathrm{~mm}$ diameter, four of 86 patients suffered acute stent occlusion, whereas in the 132 patients in whom all stents were at least $3.5 \mathrm{~mm}$ diameter there were no cases of stent occlusion $(p=0.02)$. In the unstable angina group two of 55 patients suffered acute stent occlusion compared to two of 163 patients in the stable angina group (NS). In patients with unstable angina and at least one stent of $3.0 \mathrm{~mm}$ diameter, the acute occlusion rate was $7.1 \%$ (two of 28 patients). Three of the four patients with stent occlusion had undergone complex procedures. Twenty eight patients were restudied for recurrent symptoms during the follow up period. Of these, eight patients had restenosis within their stent. In seven of these patients the stent size was $3.0 \mathrm{~mm}$ diameter, and in the remaining patient the stent size was $4.0 \mathrm{~mm}$ diameter. Three of the 28 patients restudied had developed new disease remote from the stented site, and 17 had patent stents and no significant other coronary lesion.
Conclusions-This study suggests that coronary intervention using the Multilink stent is safe and effective using aspirin alone, without IVUS or QCA, when stent diameter is greater than $3.0 \mathrm{~mm}$. All cases of stent occlusion in this series occurred in patients in whom at least one stent was $3.0 \mathrm{~mm}$ diameter, with stent occlusion being higher in patients with unstable angina compared to those with stable angina. Additional antiplatelet therapy may be beneficial in those patients in whom Multi-link stent diameter is less than $3.5 \mathrm{~mm}$, particularly in those with unstable angina, but is not necessary for patients receiving Multi-link stents of $3.5 \mathrm{~mm}$ diameter or greater.

(Heart 1998;80:499-504)

Keywords: intracoronary Multi-link stents; aspirin; stent occlusion

Use of intracoronary stents for the treatment of occlusive coronary artery disease has increased greatly over the last decade. In 1996, 46\% of all coronary angioplasty procedures undertaken in the UK involved the use of a stent, and the stent implantation rate in some centres now exceeds $80 \%$ (HH Gray, personal communication). Intracoronary stents reduce the need for emergency coronary artery bypass grafting (CABG) following abrupt vessel closure during balloon angioplasty. ${ }^{12}$ Stents also reduce the incidence of restenosis compared with simple balloon angioplasty. ${ }^{3}$ Their use, however, may be associated with unwanted effects. The most potentially serious of these is subacute thrombosis within the stent. This may occur unpredictably any time between two and 21 days after the procedure, ${ }^{5}$ and may result in acute myocardial infarction (MI) or death. The potential risk of subacute thrombosis prompted the use of aggressive anticoagulation and antiplatelet regimens in early series of patients with intracoronary stents. This resulted in significant morbidity and mortality caused by haemorrhagic complications, especially at the vascular access site, and consequently a more prolonged stay in hospital. ${ }^{34}$ In the first series of patients treated with intracoronary stents, intracoronary thrombolysis (urokinase) was added to an already aggressive anticoagulation and antiplatelet regimen. ${ }^{6}$

As experience with stent implantation has increased, it has become clear that formal anticoagulation is not required for patients undergoing intracoronary stenting, and that optimal stent placement and antiplatelet therapy are major determinants of early stent patency. ${ }^{7-9}$ 
Consequently, the majority of centres now use antiplatelet therapy alone, usually with a combination of aspirin and ticlopidine..$^{7-9}$ The incidence of subacute stent thrombosis in patients treated with aspirin and ticlopidine varies between $0.8-1.6 \% .^{7-9}$ Ticlopidine has been associated with significant neutropenia in about $2.4 \%$ of patients, ${ }^{10}$ and does not reach maximum therapeutic effect for 5-10 days after administration. ${ }^{11}{ }^{12}$ Additionally, it is not available for routine use in some countries, including the UK.

We had early experience of the Multi-link (Guidant, Basingstoke, UK) stent as a participating centre in the west European stent (WEST) trial. ${ }^{13}$ The favourable characteristics of this new stent design, ${ }^{14}$ including those of flexibility, optimal deployment, and radial strength, combined with the favourable reported experience of stent implantation without formal anticoagulation, ${ }^{7-9}$ prompted a change in our anticoagulation policy for patients receiving intracoronary stents to treatment with aspirin as a single antiplatelet agent. In this study we have prospectively monitored procedural outcome, complications, and follow up in patients who underwent treatment with Multi-link stents using postprocedural aspirin alone. Patients were divided into those in whom at least one stent was $3.0 \mathrm{~mm}$ diameter, and those in whom all stents were at least $3.5 \mathrm{~mm}$ diameter. In addition patients were stratified into those with stable and unstable angina. Unstable angina was defined as requiring treatment with intravenous heparin and glyceryl trinitrate (GTN).

\section{Patients and methods}

We prospectively monitored the outcome in 218 patients treated with 297 Multi-link stents implanted between November 1995 and March 1997. The case records and angiograms were reviewed and the patients were contacted by telephone to determine their symptoms at follow up.

Data were collected on age, sex, cardiovascular risk factors, indication for stenting, lesion(s) treated, maximum balloon inflation pressure within the stent, final balloon size, length of stent, length of hospital stay, and symptoms at follow up. At the interventional procedure the angioplasty equipment and the inflation pressures used were at the discretion of the six individual operators. Neither intravascular ultrasound (IVUS) nor quantitative coronary angiography (QCA) were used to assess vessel size or to guide stent deployment, nor were they used to assess the adequacy of the end result. All six operators judged stent deployment to be satisfactory based solely on the visual assessment of the angiographic appearance.

We examined major procedural complications and length of hospital stay. Complications at less than 30 days were defined as death, MI, reintervention or CABG, and local femoral artery complications. Complications out of hospital were assessed by telephone interview with the patients. Particular reference was made to the patients' angina status, medical therapy, and to any further hospital admissions or reintervention that occurred after discharge following their original stent insertion.

All patients were treated with $300 \mathrm{mg}$ aspirin on the day of the procedure and with heparin (10-15 $000 \mathrm{iu})$ and diazepam (10 mg) intraarterially during the procedure. Routine assessment of activated clotting time was not made during or after the procedure. On leaving the catheter laboratory patients received aspirin, either $150 \mathrm{mg}$ or $300 \mathrm{mg} /$ day; systolic blood pressure was rigorously maintained at $110 \mathrm{~mm} \mathrm{Hg}$ or greater using intravenous fluid replacement if required. The majority of patients $(\mathrm{n}=186 ; 85 \%)$ received no further heparin and the arterial sheath was removed four hours after the procedure with external compression; 32 patients (15\%) were treated overnight with intravenous heparin $(1000 \mathrm{iu} /$ h). The decision to continue treatment with heparin was not influenced by lesion complexity, stent size, stent length, or the presence of unstable angina, but was purely practical to avoid femoral sheath removal at night. Ticlopidine was not used in any patient either alone or in addition to aspirin therapy, nor did any patient in the study receive other IIb/IIIa inhibitors.

\section{Results}

A total of 218 patients were included in the study, in whom 297 Multi-link stents (Guidant) were implanted to treat 266 coronary lesions. The Multi-link stents used were $15 \mathrm{~mm}, 25 \mathrm{~mm}$, or $35 \mathrm{~mm}$ long and were premounted on a rapid exchange PE 600 balloon (Guidant) with no stent delivery system. The average age of the patients was 60.4 years (range $37-82$ years) and 166 were male (76\%) (table 1).

Of the 218 patients treated, $173(79 \%)$ had stent implantation to a single vessel, and of these 40 had more than one stent placed in the target vessel. Forty five patients $(21 \%)$ had multivessel intracoronary intervention; of these 21 had multivessel stent implantation and the remainder had single vessel stent implantation with additional balloon angioplasty to a further vessel (fig 1). Two hundred and five patients $(87 \%)$ were on long term aspirin before the procedure (75-300 $\mathrm{mg}$ ), and 55 patients $(25 \%)$ had unstable angina requiring treatment with intravenous heparin and GTN at the time of their coronary intervention. The vessels treated and the lesion characteristics (American Heart Association/

Table 1 Patient characteristics

\begin{tabular}{ll}
\hline Total patients & 218 \\
Average age & 60.4 years \\
Age range & $37-82$ years \\
Male & $166(76 \%)$ \\
Unstable angina & $55(25 \%)$ \\
Hypercholesterolaemia & $116(53 \%)$ \\
Current smoker & $31(14 \%)$ \\
Ex-smoker & $122(56 \%)$ \\
Hypertension & $76(35 \%)$ \\
Diabetes & $19(9 \%)$ \\
Family history & $123(56 \%)$ \\
Previous MI & $91(42 \%)$ \\
Total lesions & 266 \\
Total stents & 297
\end{tabular}



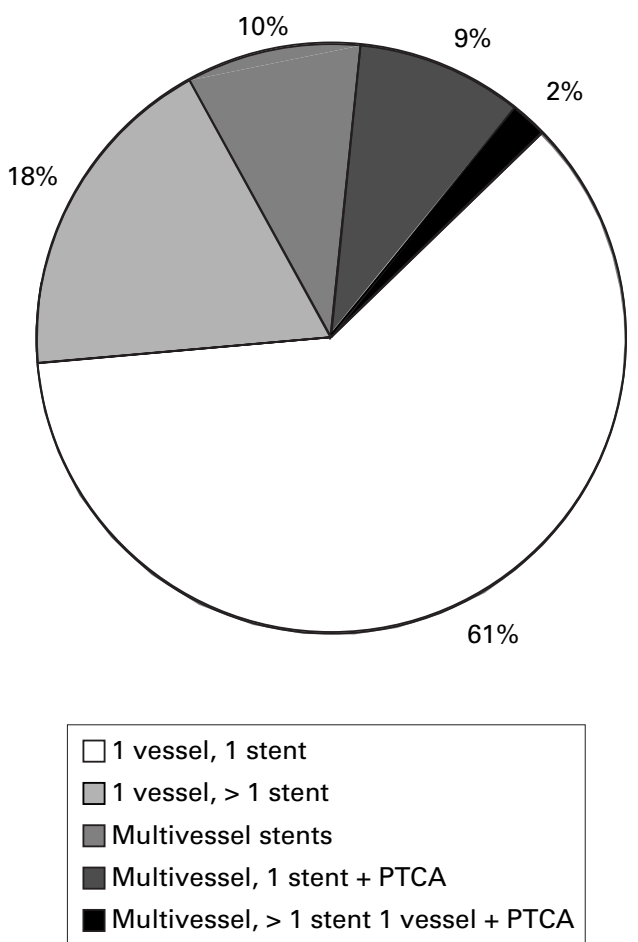

Figure 1 Proportion of single and multivessel interventions in 218 patients.

Table 2 Distribution of vessels treated and the lesion characteristics (American Heart Association/American College of Cardiology criteria)

\begin{tabular}{llllll}
\hline \multicolumn{5}{c}{ Lesion characteristics } \\
\cline { 2 - 5 } Vessel & $A$ & $B 1$ & $B 2$ & $C$ & Total (\%) \\
\hline LAD & 45 & 45 & 18 & 4 & $112(42)$ \\
Cx & 11 & 20 & 7 & 4 & $42(16)$ \\
RCA & 20 & 39 & 28 & 8 & $95(36)$ \\
D1 & 1 & 1 & 1 & & $2(0.7)$ \\
Ix & 1 & & & & $1(0.4)$ \\
L main & 1 & 3 & 3 & 7 & $1(0.4)$ \\
SVG & & 3 & $3(5)$ & $13(5)$ \\
Total (\%) & $78(29)$ & $108(41)$ & $57(21)$ & $23(9)$ & 266
\end{tabular}

LAD, left anterior descending; $\mathrm{Cx}$, circumflex; RCA right coronary artery; D1, first diagonal; Ix, intermediate; L main, left main; SVG, saphenous vein graft.

American College of Cardiology criteria) are shown in table 2. The indications for stenting and the original lesion characteristics are shown in table 3, and the size and length of stents used are shown in table 4 .

Indications for stenting were as follows: elective implantation 197 (74\%); suboptimal result after balloon angioplasty $54(20 \%)$; bailout 9 $(3 \%)$; and restenosis at previous angioplasty site $6(2 \%)$. Predilatation was performed in 216 patients $(99 \%)$. No debulking procedures were performed in any of the patients. All operators aimed to cover the lesion completely with the

Table 3 Indications for stenting and the lesion characteristics (American Heart Association/American College of Cardiology criteria) of the vessels stented

\begin{tabular}{llllll}
\hline \multirow{5}{*}{ Stent indication } & \multicolumn{2}{l}{ Lesion characteristics } & \\
\cline { 2 - 5 } & $A$ & $B 1$ & $B 2$ & $C$ & Total (\%) \\
\hline Elective & 52 & 82 & 47 & 16 & $197(74)$ \\
Suboptimal & 23 & 19 & 10 & 2 & $54(20)$ \\
Bailout & 2 & 2 & & 5 & $9(3)$ \\
Restenosis & 1 & 5 & $57(21)$ & $23(9)$ & 266 \\
Total (\%) & $78(29)$ & $108(41)$ & & & \\
\hline
\end{tabular}

Table 4 Size and length of stent used

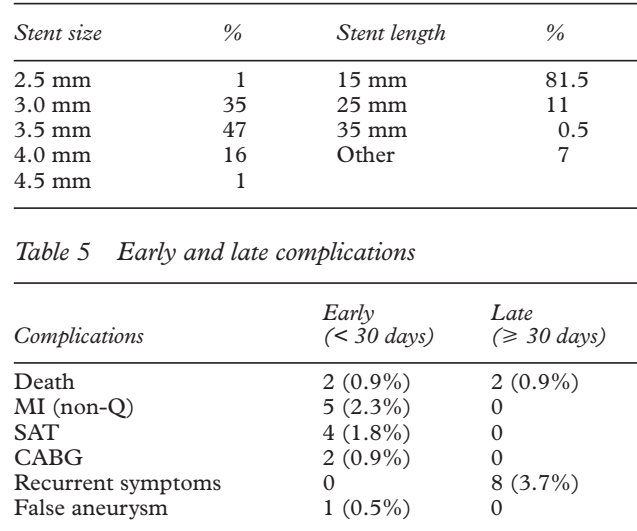

MI, myocardial infarction; SAT, subacute stent thrombosis; $\mathrm{CABG}$, coronary artery bypass grafting.

stent. Moderate high pressure inflation was used in all patients for initial deployment of the stents, and high pressures were used in all patients for either initial implantation or further postimplantation dilatation, the maximum pressure (SD) being 14 (1.9) atmospheres.

The mean (SD) postprocedure length of hospital stay was 2.0 (2.1) days; 119 patients $(55 \%)$ were discharged the day after the procedure, 62 patients $(28 \%)$ were discharged after two days, and 18 patients ( $8 \%$ ) after three days.

\section{COMPLICATIONS WITHIN 30 DAYS}

There were two deaths $(0.9 \%)$ within the first 30 days of follow up. Two further patients suffered subacute stent thrombosis $(0.9 \%)$, both of whom were successfully treated with emergency CABG. Non-Q wave MI occurred in five patients $(2.3 \%)$, and one further patient developed a false aneurysm of the femoral artery that required surgical repair (table 5). Thus total event free survival within the first 30 days (freedom from death, CABG, acute MI, reintervention) was $94.9 \%$, and ranked event free survival was $95.9 \%$.

Of the four cases of acute stent occlusion, three occurred in patients with complex coronary lesions, in whom multiple stents or long (greater than $15 \mathrm{~mm}$ ) stents had been used. Of the two patients who died, one had a $15 \mathrm{~mm}$ long, $3.0 \mathrm{~mm}$ diameter stent placed electively in the left anterior descending (LAD) artery. This occluded on the night of the procedure and the patient died in the catheter laboratory during attempts to reopen the vessel. The other patient who died had a $15 \mathrm{~mm}, 3.0 \mathrm{~mm}$ stent placed in the atrioventricular circumflex and a $25 \mathrm{~mm}, 3.5 \mathrm{~mm}$ stent placed in the right coronary artery. She died suddenly at home four days after the procedure. Postmortem examination showed that both stents had occluded.

Of the two remaining cases of acute occlusion, one was a bifurcation lesion involving the LAD and first diagonal, in which a stent was placed in the LAD across the origin of the diagonal and a further stent was then placed in the diagonal through the side of the LAD stent in a "trouser" formation. In view of the 
Table 6 Stratification of patients according to angina status and stent size

\begin{tabular}{lrl}
\hline & $n$ & Occlusion $n(\%)$ \\
\hline Stable angina, size $\leqslant 3.0 \mathrm{~mm}$ & 58 & $2(3.4)$ \\
Unstable angina, size $\leqslant 3.0 \mathrm{~mm}$ & 28 & $2(7.1)$ \\
Stable angina, size $\geqslant 3.5 \mathrm{~mm}$ & 105 & $0(0)$ \\
Unstable angina, size $\geqslant 3.5 \mathrm{~mm}$ & 27 & $0(0)$ \\
\hline
\end{tabular}

complex nature of the intervention this patient had an elongated postprocedure stay in hospital and suffered subacute thrombosis in the LAD stent three days after the procedure. The LAD and diagonal were occluded with obvious thrombus within the stents. The vessels were reopened and the patient underwent successful CABG to both vessels. He left hospital seven days after his surgery.

In the other case, a $25 \mathrm{~mm}, 3.0 \mathrm{~mm}$ stent was deployed to "bailout" a long spiral dissection in the LAD with acute LAD occlusion that occurred as a result of angioplasty. After the insertion of the stent to cover the proximal dissection, it was apparent that there was further dissection distally that appeared separate from the stented area. It was elected to treat this conservatively as there was TIMI 3 flow angiographically. This patient suffered subacute stent thrombosis 36 hours later. The $\mathrm{LAD}$ was found to be occluded at the proximal stent with obvious thrombus formation. The vessel was reopened and the patient underwent successful CABG with a left internal mammary artery graft to the LAD. He left hospital nine days after his surgery.

Three further patients were restudied during the first 30 days after stent insertion because of recurrent chest pain. All three had widely patent stents with no other significant coronary disease.

Patients were stratified according to stent size $(3.0 \mathrm{~mm}$ diameter or less, or $3.5 \mathrm{~mm}$ diameter or more), and angina status (stable or unstable) (table 6). In the unstable angina group two of 55 patients suffered acute occlusion $(3.6 \%)$, compared to two of $163(1.2 \%)$ patients in the stable angina group (NS). In patients in whom at least one stent was $3.0 \mathrm{~mm}$ diameter, four of 86 patients $(4.7 \%)$ suffered acute occlusion. In 132 patients in whom all stents were at least $3.5 \mathrm{~mm}$ diameter there were no cases of stent occlusion $(p=0.02)$. In patients with unstable angina and at least one stent equal to $3.0 \mathrm{~mm}$ diameter, the acute occlusion rate was $7.1 \%$ (two of 28 patients). In those patients with stable angina and at least one stent equal to $3.0 \mathrm{~mm}$ diameter the acute occlusion rate was $3.4 \%$ (two of 58 patients). There was no significant difference in stent occlusion between patients treated with a single stent and those who had multiple stents inserted, nor between patients who had single vessel intervention compared with those who underwent multivessel intervention.

FOLLOW UP

Follow up data were obtained in 217 patients $(99.5 \%)$ by telephone interview with particular reference to their angina status, medical therapy, and to any further hospital admissions that occurred after hospital discharge from their initial stent insertion, or any reintervention. One patient was lost to follow up, having moved from his previous address. The mean (SD) length of follow up was 293 (122) days after the stent insertion. The minimum time to follow up was 46 days. Follow up was greater than 100 days in $96 \%$ of the patients, and greater than 180 days in $79 \%$. During this period there were two deaths at 101 days and 137 days after the stent, respectively. There were no other major cardiac events (CABG, acute MI).

When questioned about their angina symptoms, $76 \%$ of the patients were completely free from angina and a further $14 \%$ found that their angina had greatly reduced following stent implantation, such that angina did not limit their activities. Twenty five patients $(11.5 \%)$ were restudied because of recurrent chest pain, with restudy occurring at a mean of 5.6 months of follow up (range 2-15 months). Of these, eight patients had major (greater than 50\%)

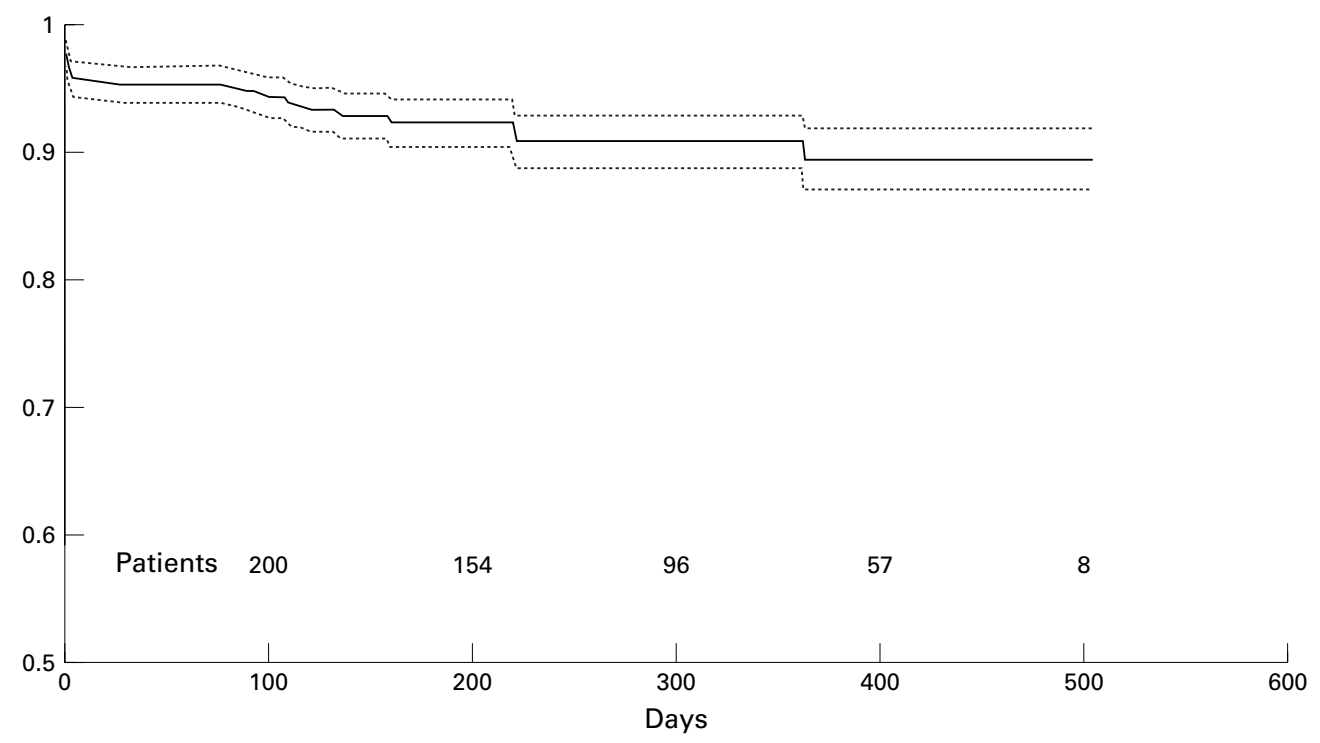

Figure 2 Event free survival in 218 patients over the follow up period. Data are shown $\pm 70 \%$ confidence intervals. 
restenosis within their stent, six of whom were treated with further angioplasty, one was referred for CABG, and one was treated medically. In seven of the eight patients with restenosis the original stent size was $3.0 \mathrm{~mm}$ diameter or less $(p=0.02)$. In three patients there was other native disease progression, with the site of stent implantation being widely patent. In 14 patients the stent site was widely patent and there was no other significant coronary disease. Hence recurrent angina as a consequence of target lesion restenosis occurred in $3.7 \%$ of the patients during the follow up period. Thus, event free survival (freedom from death, CABG, acute MI, re-intervention) over the follow up period from the initial procedure was $89.8(2.4) \%(70 \%$ confidence intervals) (fig 2).

\section{Discussion}

It has been suggested that intracoronary stenting is safe with antiplatelet therapy alone if IVUS is used to guide stent placement. ${ }^{71516}$ Data from the WEST I trial, ${ }^{13}$ using the same Multi-link stent but an aggressive anticoagulation regimen, had already suggested that subacute thrombosis rates would be likely to be low with the Multi-link stent, since the rate of stent occlusion in the WEST I trial was $1 \%$. Furthermore, there were no cases of stent thrombosis in the aspirin alone group (126 patients) in the WEST II trial, in which the Multi-link stent was used with QCA and IVUS to ensure optimal stent deployment (PW Serruys, personal communication).

This is an observational study of the effect of a change of practice in the management of patients undergoing intracoronary stent insertion at one tertiary cardiology referral centre in the UK. In our study neither IVUS nor QCA were used and the overall stent occlusion rate $(1.8 \%)$ was comparable to that in other studies, ${ }^{7}{ }^{15} 16$ and complications within 30 days in our patients remained low (table 5). Antiplatelet therapy was provided using aspirin alone, without the addition of ticlopidine. Ticlopidine was not used because of concern over possible bone marrow toxicity necessitating routine checks on haematology, ${ }^{10}$ and because it is available in the UK on a named patient basis only.

We believe that our data provide evidence that, following intracoronary stent implantation using the Multi-link stent, treatment with aspirin alone, without additional IVUS or QCA, is sufficient to prevent acute occlusion provided the stent size is $3.5 \mathrm{~mm}$ or greater. Such an approach has the advantage of being simple and cost effective, and avoids exposing patients to the potentially serious adverse effects of ticlopidine, particularly bone marrow suppression.

All cases of stent occlusion in our series occurred in patients in whom at least one stent was $3.0 \mathrm{~mm}$ diameter. Patients with unstable angina were at particular risk. We suggest that adjuvant methods for assessing adequate stent deployment, such as IVUS and QCA, may be reserved for those patients in whom stent size is $3 \mathrm{~mm}$ diameter or less. In these same patients additional antiplatelet therapy with ticlopidine or other oral IIb/IIIa inhibitors may be beneficial in preventing stent thrombosis.

Patients with unstable angina have an increased risk of adverse events following coronary intervention. ${ }^{16}$ In our series the rate of stent occlusion was increased threefold in patients with unstable angina compared to those with stable angina, and was particularly high in those patients with unstable angina in whom at least one stent was $3.0 \mathrm{~mm}$ diameter $(7.1 \%)$. These patients are especially vulnerable to stent thrombosis, and although the number of patients in this group is small in our study ( $n=28$ ), our observations suggest that these patients cannot be treated safely with aspirin alone.

We are not able to assess whether the addition of ticlopidine would have affected the outcomes we have observed in our group of patients. Hall and colleagues, ${ }^{17}$ using IVUS guided stent deployment, randomised 226 patients to treatment with aspirin alone or aspirin and ticlopidine following stenting. They found that neither the stent thrombosis rate $(2.9 \%)$ nor the incidence of $\mathrm{MI}$ at one month $(3.9 \%)$ was significantly different in the aspirin alone group from that in the aspirin and ticlopidine group $(0.8 \%$ and $0.8 \%$, respectively). This study has insufficient power to exclude a real difference in favour of the addition of ticlopidine. Preliminary data from the STARS investigators, soon to be published fully, supports the suggestion that the addition of ticlopidine may confer benefit, at least as far as the Palmaz-Schatz stent is concerned, because the reported stent occlusion rates were $2.9 \%$ in the aspirin alone group $v 0.6 \%$ in the aspirin and ticlopidine group. ${ }^{18}$ The STARS study comprised patients with single vessel disease in whom stents were placed electively. In our study the overall stent occlusion rate was $1.8 \%$, and if patients with single vessel intervention only are considered, the occlusion rate falls to $1.1 \%$. These occlusion rates, which include higher risk patients with unstable angina, are $35-60 \%$ lower than those reported in the STARS trial. This difference may result from the different stent designs used in the two studies. Preliminary data from the ACS Multilink stent clinical equivalence in de novo lesions trial (ASCENT), comparing the Multilink stent to the Palmaz-Schatz stent in a randomised trial of 1040 patients, suggests that subacute stent thrombosis is less common in patients receiving a Multi-link stent $(0.4 \% v$ $1.9 \% ; \mathrm{p}=0.04) .{ }^{19}$ Furthermore, no data are available as to stent diameter in the STARS (stent anticoagulation regimen study) cohort of patients. Our series suggests that occlusion is more frequent when stent size is $3.0 \mathrm{~mm}$ diameter but less common when stents $3.5 \mathrm{~mm}$ diameter or greater are used.

Experimental data suggest that stent design may influence vascular injury at the time of implantation as well as the subsequent likelihood of thrombosis and restenosis. ${ }^{14}$ Most of the published series relate to first generation stents, such as Palmaz-Schatz, which may not be applicable to newer stent designs. This, 
coupled with increased awareness of the need for optimal stent deployment, may be more important as a determinant of stent related complications than the choice of antiplatelet agents. It is unclear whether the results of this study are stent specific, although it seems more likely that a strategy of aspirin alone will be more suitable for second generation stent designs than their original counterparts.

It is known that some individuals do not respond to or are genuinely allergic to aspirin, and it seems likely that an alternative antiplatelet agent will need to be sought for this group of individuals. A possible candidate drug is clopidogrel which inhibits ADP dependent activation of the GPIIb/IIIa receptor, a mechanism of action similar to ticlopidine, although its onset of action is quicker than that of ticlopidine. ${ }^{20}$ Clopidogrel has recently been shown to be as effective as aspirin in reducing the combined risk of ischaemic stroke, MI, or vascular death in patients with recent ischaemic stroke, $\mathrm{MI}$, or symptomatic peripheral arterial disease. ${ }^{21}$ The overall safety profile of clopidogrel appears to be at least as good as that of aspirin, ${ }^{21}$ although there have been reports of significant reductions in neutrophils associated with its use. ${ }^{21}$ Other possible candidate drugs are the various oral IIb/IIIa receptor antagonists currently being investigated in clinical trials-for example, xemilofiban in the EXCITE study (efficacy and safety of xemlofiban administration to patients undergoing coronary angioplasty or stent placement).

The use of antiplatelet therapy alone after stent placement has the advantage of allowing a much shorter hospital stay. In our study $55 \%$ of patients were discharged from hospital within 24 hours of the stent procedure, and the mean length of postprocedure hospital stay for the series was 2.0 days. This reduced hospital stay has the advantage of reducing hospital costs, and our data suggest that this is achieved without a detrimental effect on patient outcome. In addition, costs were further reduced because we did not use QCA or IVUS. This approach had no apparent adverse effect on outcome in patients in whom stent size was $3.5 \mathrm{~mm}$ or greater since there were no acute occlusions in this group. The overall rate of subacute thrombosis that we observed is comparable to that seen in other studies ${ }^{7-9} 15$; however, all these occurred in patients with at least one $3.0 \mathrm{~mm}$ diameter stent.

Our study suggests that coronary intervention using the Multi-link stent is safe and effective using a simple and cost effective approach with aspirin alone in patients in whom stent size is at least $3.5 \mathrm{~mm}$ diameter. It is possible that the additional use of supplementary antiplatelet therapy, IVUS, and QCA might confer further benefit in those cases involving smaller (3.0 mm diameter) stents, particularly in patients with unstable angina.

1 Kuntz RE, Piana R, Pomerantz RM, et al. Changing incidence and management of abrupt closure following coronary intervention in the new device era. Cath Cardiovasc Diag 1992;27:183-90.

2 Roubin G, Cannon AD, Agrawal S, et al. Intracoronary stenting for acute and threatened closure complicating transluminal coronary angioplasty. Circulation 1992;85: 916-27.

3 Serruys PW, de Jaegere P, Kiemeneij F, et al. A comparison of balloon-expandable-stent implantation with balloon angioplasty in patients with coronary artery disease. angioplasty in patients with coronary artery disease.
Benestent study group. $N$ Engl f Med 1994;331:489-95.

4 Fischmann DL, Leon MB, Baim DS, et al. A randomised comparison of coronary-stent placement and balloon angioplasty in the treatment of coronary artery disease. Stent restenosis study investigators. N Engl f Med 1994;331:496 501 .

5 Savage MP, Fischmann DL, Schatz RA, et al. Long-term angiographic and clinical outcome after implantation of a balloon-expandable stent in the native coronary circulation. Palmaz-Schatz Stent Study Group. $\mathcal{f}$ Am Coll Cardiol 1994;24:1207-12.

6 Sigwart U, Puel J, Mirkovitch V, et al. Intravascular stents to prevent occlusion and restenosis after transluminal angioplasty. N Engl f Med 1987;316:701-6.

7 Colombo A, Hall P, Nakamura S, et al. Intracoronary stenting without anticoagulation accomplished with intravascu-
lar ultrasound guidance. Circulation 1995;91:1676-88.

8 Morice MC, Breton C, Bunouf $\mathrm{P}$, et al. Coronary stenting without anticoagulant, without intravascular ultrasound. without anticoagulant, without intravascular ultrasound.

9 Schomig A, Neumann FJ, Kastrati A, et al. A randomised comparison of antiplatelet and anticoagulant therapy after the placement of coronary-artery stents. $N$ Engl f $\mathrm{Med}$ 1996;334:1084-9.

10 Ticlopidine and white blood cell disorders. In: Current problems in pharmacovigilance. London: Medicines Control Agency, 1997;23:2.

11 Harker LA, Bruno JB. Ticlopidine's mechanism of action on human platelets. In: Hass WK, Easton JD, eds. Ticlopidine, platelets and vascular disease. New York: Springer-Verlag, 1993:41-59.

12 Gregorini L, Marco J, Fajadet J, et al. Ticlopidine and acetylsalicylic acid pre-treatment reduce coagulation and platelet activation during coronary dilatation procedures. $\mathcal{F}$ Am Coll Cardiol 1997;29:13-20.

13 van der Giessen W, Emanuelsson H, Dawkins K, et al. Clinical and angiographic results with the Multi-link ${ }^{\mathrm{TM}}$ coronary cal and angiographic results with the Multi-link coronary
stent system. The west European stent trial (WEST) stent system. The west European stent trian
[abstract]. Eur Heart $\mathcal{F} 1996 ; 17($ suppl 22):19.

14 Rogers C, Edelman ER. Endovascular stent design dictates experimental restenosis and thrombosis. Circulation 1995; 91:2995-3001.

15 Blasini R, Neumann FJ, Richardt G, et al. Intravascular ultrasound-guided emergency Palmaz-Schatz stent placement without post-procedural systemic anticoagulation. Heart 1996;76:344-9.

16 de Feyter PJ, van den Brand M, Laarman GJ, et al. Acute coronary artery occlusion during and after percutaneous transluminal coronary angioplasty: frequency, prediction, clinical course, management, and follow-up. Circulation 1991;83:927-36.

17 Hall P, Nakamura S, Maiello L, et al. A randomised comparison of combined ticlopidine and aspirin therapy versus aspirin therapy alone after successful intravascular ultrasound-guided stent implantation. Circulation 1996;93: 215-22.

18 More R, Chauhan A. Antiplatelet rather than anticoagulant therapy with coronary stenting. Lancet 1997;349:146-7.

19 Baim DS, Cutlip DE, Midei M, et al. ACS Multi-link ${ }^{\mathrm{TM}}$ stent clinical equivalence in de novo lesions trial (ASCENT) [abstract]. Circulation 1997;96:I-593.

20 Savi P, Herbert JM. ADP receptors on platelets and ADP selective antiaggregating agents. Med Res Rev 1996;16:159-

21 CAPRIE Steering Committee. A randomised, blinded, trial of clopidogrel versus aspirin in patients at risk of ischaemic events (CAPRIE). Lancet 1996;348:1329-39. 